\title{
4 Üben als reflexive Praxis und Übeangebote im Rahmen von optes
}

Gabi Reinmann, Christian Schmidt, Victoria Marquardt, Daria Paul

\subsection{Einführung}

An vielen Hochschulen werden mathematische Brückenkurse angeboten, um Studierenden den Übergang von der Schule zur Hochschule zu erleichtern. Sie sollen in diesen Angeboten in der Regel Defizite identifizieren und ausgleichen sowie mathematische Inhalte auffrischen und festigen. Während dieses Ziel flächendeckend zu gelten scheint, ist die didaktische Konzeption der Brückenkurse durchaus unterschiedlich.

Digitale Medien spielen in vielen Angeboten eine zentrale Rolle (Bausch et al. 2014), da sie geeignete Merkmale bzw. Funktionen für das Üben umfassen. Übungsprogramme (zunächst Computer-Based Trainings, später Web-Based Trainings) mit automatischen Feedback-Funktionen und diversen Individualisierungsmöglichkeiten haben von jeher vor allem Effizienzvorteile versprochen. Heute finden sich auf diesem Gebiet reine Online-Varianten ebenso wie Blended-LearningAngebote und in vielen Fällen gibt es Koppelungen mit Self-Assessment-Möglichkeiten (Greefrath et al. 2015).

Die weite Verbreitung von Brückenkursen, die erkannte Relevanz und zahlreiche praktische Lösungsversuche unter Nutzung digitaler Medien stehen aus einer hochschuldidaktischen Perspektive in einem deutlichen Missverhältnis zur verfügbaren Theorie und Empirie des Übens als einer Lernform, die auch an Hochschulen ihre Bedeutung und Berechtigung hat. Theoretisch sind allgemeindidaktische und lerntheoretische Bestimmungen des Lernens in der Regel unterkomplex: Üben wird als sekundär betrachtet und dem Lernen gegenübergestellt, während die Möglichkeit, Üben als eine elementare Lernform zu betrachten, in vielen Fällen außen vor bleibt (Brinkmann 2012). Empirisch konzentrieren sich Studien und Erkenntnisse auf spezielle Domänen (z. B. Musik, Bewegung, Lesen, Schreiben, Rechnen) sowie auf die Schule - oft begleitet vom schlechten Ruf des Übens als Pauken, Drill-and-Practice oder Maßnahme der Disziplinierung. Anzunehmen ist des Weiteren, dass man das Üben als Lernform an Hochschulen im Zuge konstruktivistischer Ansätze schlichtweg aus den Augen verloren hat.

Die Originalversion dieses Kapitels wurde revidiert. Ein Erratum ist verfügbar unter https://doi.org/10.1007/978-3-658-31279-4_32 


\section{2 Üben als elementare Form des Lernens}

Die Anzahl der Autor*innen, die sich mit dem Üben aus einer allgemeindidaktischen Perspektive beschäftigen, ist überschaubar, hat sich über die Jahrzehnte nur wenig vergrößert und ist weitgehend auf die Schule beschränkt geblieben. Eine frühe Beschäftigung mit dem Üben liefert Bollnow (1978). Er plädiert dafür, beim Üben nicht nur den äußeren Effekt im Blick zu haben (Üben als Mittel zum Zweck), sondern dem Akt des Übens als solchen, der damit verbundenen Konzentration und Sorgfalt, aber auch der (potenziell möglichen) Freude am Prozess Aufmerksamkeit zu schenken (Üben als Selbstzweck).

Aebli hat in den 1980er Jahren das Üben ebenfalls aufgegriffen und es zu einer der Grundformen des Lehrens erklärt (Aebli 2006). Ähnlich wie Bollnow entkräftet er die Kritik am Üben als sinnentleert und ineffektiv, indem er betont, dass es keineswegs nur um den Aufbau von Routinen, sondern um ein Verstehen durch variable Übungsmöglichkeiten gehe. Auch Prange (2005) sieht im Üben eine zentrale „Operation des Lernens“, mit der Fähigkeiten und Fertigkeiten aufgebaut werden.

Brinkmann $(2011,2012)$ geht (auch methodisch) über die genannten pädagogischen und lernpsychologischen Vorstellungen hinaus. Er macht neben anderen Aspekten auf die Selbstbildung und Übung als Lebenskunst aufmerksam. „Üben (als Prozess) ist Ausüben (einer Sache) im Einüben (einer Fähigkeit) bei gleichzeitigem Sich-selbst-üben“" (Brinkmann 2012, S. 38). In diesem Sinne Üben als Praxis (gegebenenfalls auch als akademische Praxis) zu begreifen und die Persönlichkeitsbildung einzubeziehen, scheint in hohem Maße kompatibel mit dem Anspruch akademischen Lehrens und Lernens (Prenzel 2015). Die weiteren Ausführungen lehnen sich daher überwiegend an Brinkmann (2012) und seine phänomenologische und historisch-rekonstruktive Analyse des Übens an, in der Erwartung, damit den Bogen zur akademischen Lehre zu schlagen und ein Bindeglied zwischen hochschul- und fachdidaktischen Anforderungen zu entwerfen.

\subsubsection{Konstituierende Merkmale des Übens}

Üben zielt darauf $a b$, handlungsrelevante Fähigkeiten und Fertigkeiten aufzubauen, um etwas zu können bzw. besser zu können (als zuvor). Geübt wird, wenn man eine angestrebte Fertigkeit oder Fähigkeit (noch) nicht beherrscht, scheitert und es erneut versucht. Eine grundlegende Voraussetzung für das Üben ist, dass Übende eigene Erwartungen an ihr Handeln haben, weil sie es nur dann als unzulänglich erleben können. Scheitern resultiert aus der Enttäuschung einer Selbsterwartung und ist vermutlich ein wichtiges Motiv zum Üben (Brinkmann 2012). „Die Negativität im Üben - die Erfahrung von Enttäuschung, Irritation, Verfehlen, Vergessen, Scheitern - gehört somit elementar zum Üben hinzu“ (Brinkmann 2012, S. 396). Die übende Person braucht entsprechend die Einstellung, eigenes 
Ungenügen bzw. Nicht-Können auszuhalten. Genau dies könnte der entscheidende erste Schritt zur Motivation sein, etwas zu üben.

Unabhängig davon, was man übt, besteht das Ziel in der Regel darin, das, was man übt, am Ende besser zu können, zu perfektionieren oder vollkommener zu machen. Das Primat des Könnens (Brinkmann 2012) ist demnach ein weiteres zentrales Merkmal des Übens. Es hat Schnittstellen zu anderen didaktischen Konzepten, vor allem zur „Meisterlehre“ (Cognitive Apprenticeship: Collins, Brown und Newman 1989) und zum ,gezielten Training“ (Deliberate Practice: Ericsson, Krampe und Tesch-Römer 1993).

Ein weiteres, mit dem Primat des Könnens eng verwobenes Merkmal des Übens ist die Wiederholung. „Etwas zu wiederholen bedeutet weder eine Iteration völlig identischer Elemente noch eine Wiederkehr von gänzlich Verschiedenem“ (Brinkmann 2012, S. 39). Phänomenologische Analysen des Übens (ebd.) stellen das Wiederholen in ein anderes Licht als es psychologische Analysen tun. Während es in der Lernpsychologie darum geht, durch Wiederholung Wissensstrukturen zu festigen und/oder zu automatisieren und prozedurales Wissen zu generieren (Anderson 1993), hebt die Phänomenologie zum einen den Spielraum des Übens hervor, der sich durch wiederholende Aktivitäten ergibt, und betont zum anderen den Widerstreit zwischen dem Willen, etwas zu beherrschen (Können), und der Erkenntnis der damit verbundenen Grenzen (Scheitern). Wiederholung kann entschleunigenden (bis hin zu meditativen) Charakter annehmen und Formen des Resonanzerlebens (etwa im Einüben einer Sache) ermöglichen (Rosa 2016).

In Abgrenzung zum mechanischen Pauken verlangt das Üben als eine elementare Lernform in Bildungskontexten nach Reflexion, also danach, Übungsprozesse auch zu unterbrechen, zu bewerten und Folgerungen für das weitere Üben zu ziehen (Bollnow 1978). Das Merkmal der Reflexion und die damit verbundene Iteration (üben - scheitern - reflektieren - weiter üben) erinnert an Konzepte des Erfahrungslernens bzw. reflexiven Lernens, wie es zum Beispiel mit dem „experimential learning cycle" von David Kolb (1984) postuliert wird. Allein das TätigSein (Erfahrungen machen, experimentieren) garantiert noch kein Lernen; erst Reflexion ermöglicht es, aus dem aktivem Tun zu lernen, Begriffe zu bilden, neue Ordnungen zu erkennen und letztlich auch das Lernen, respektive Üben, zu lernen. In diese Richtung gehen etwa metakognitive Ansätze zum Selbstlernen (Friedrich und Mandl 2006). Auch hier ist Reflexion Voraussetzung dafür, dass man lernt, wie man effektiv lernt (also auch übt), unter welchen Voraussetzungen dies am besten gelingt, wie man sich hierzu motiviert und vorhandene soziale oder materiale Ressourcen nutzen kann. 


\subsubsection{Determinanten des Übens}

Wovon hängt es ab, dass Üben als ein elementarer Prozess des Lernens möglich wird und die Merkmale (Zulassen von Scheitern, Primat des Könnens, Wiederholung, Reflexion) entfaltet, die als konstituierend für das Üben gelten? Insgesamt betrachtet lassen sich dazu wenige gesicherte Aussagen machen. Im Folgenden werden einige externe und interne Determinanten für gelingendes Üben, wie man sie in der Literatur findet, aufgelistet.

$\mathrm{Zu}$ den externen Determinanten zählen in der Regel Rahmenbedingungen des Übens wie Materialien, Aufgaben und Instrumente (auch Medien) sowie Lehrende, die etwas vormachen, unterstützen oder korrigieren, und Peers bzw. Mitlernende (Brinkmann 2011). Man könnte auch in Anlehnung an die Metakognitionsforschung von materiellen und sozialen Ressourcen sprechen, die den Übenden zur Verfügung stehen, von diesen aber als solche erkannt und genutzt werden müssen, bevor sie wirksam werden. Alle Ressourcen zum Üben - so ließe sich theoretisch postulieren - sollten dafür geeignet sein, dass Lernende Kenntnisse, Fertigkeiten, Fähigkeiten oder Haltungen variierend wiederholen, aber eben auch in Hinblick auf das angestrebte Können reflektieren.

Lehrende, gegebenenfalls auch Mitlernende, können Feedback geben und den Prozess des Übens begleiten, sofern die dazu erforderlichen Fähigkeiten vorhanden sind. Wie in der „Meisterlehre“ herausgearbeitet, sind Aspekte von Coaching und Scaffolding (Collins et al. 1989) wichtige Determinanten dafür, dass Expertise durch Üben ausgebildet wird. Günstige Rahmenbedingungen für das Üben sollten in der Folge solche sein, die Lernende gezielt und möglichst individuell dabei unterstützen, die eigenen Erwartungen an ihr Können zu formulieren, im Prozess des Übens Momente des Scheiterns auszuhalten, Fehler und Unvollkommenheiten zu analysieren und daraus Motivation für weiteres Üben zu schöpfen.

Interne Determinanten gelingenden Übens sind zum einen abhängig vom Ziel und letztlich auch von den externen Bedingungen des Übens, mit denen Lernende in Interaktion treten. Zum anderen aber lassen sich wiederum theoretisch begründet einige grundlegende Voraussetzungen für ein Üben im hier gemeinten Sinne annehmen. $\mathrm{Zu}$ nennen sind zunächst einmal Einsicht und Problembewusstsein: Lernende müssen erkennen und für sich zu dem Schluss kommen, dass ihre Kenntnisse, Fähigkeiten oder Haltungen unzulänglich sind. Damit verbunden sind explizite Erwartungen an das eigene Können und die Motivation, die eigene Leistung zu steigern (Brinkmann 2011). Erst dann stellt sich auch eine Bereitschaft zum Üben ein. Konzentration und Durchhaltevermögen (auch im Falle des Scheiterns) können als weitere interne Determinanten gelingenden Übens angesehen werden. Allerdings sind Einsicht und Problembewusstsein, Selbsterwartung und Motivation sowie Konzentration und Durchhaltevermögen nicht zwangsläufig nur Voraussetzungen des Übens, sondern prinzipiell auch Folgen des Übens, die im Prozess erst entstehen und dann wachsen können (Brinkmann 2012; Meyer 1987). 


\section{3 Üben in der Mathematik}

Der Übebegriff in der Mathematik ist (anders als etwa in der allgemeinen Hochschuldidaktik) etabliert und zudem in weiten Teilen lernpsychologisch geprägt. Geübt wird, um das Gelernte zu sichern, zu vertiefen und die Fähigkeit zu entwickeln, das Gelernte in ähnlichen Situationen anzuwenden (Scherer und Weigand 2017). Man geht davon aus, dass das Üben in der Mathematik diejenigen Lerntätigkeiten umfasst, die „darauf ausgerichtet sind, neue oder schon früher kennengelernte (mathematische) Begriffe, Zusammenhänge und Verfahren sowie Vorgehensstrategien in variierenden Kontexten verfügbar zu haben und verständig verwenden zu können“ (Bruder 2008, S. 4). Das gelingt nicht durch bloßes Zuschauen oder einfaches Nachahmen von Lösungsbeispielen, sondern verlangt danach, mathematische Aufgaben selbst zu bearbeiten und dies wiederholt $\mathrm{zu}$ tun (Scherer und Weigand 2017).

In der mathematikdidaktischen Literatur findet man mehrere Versuche, Übungsformate zu unterscheiden und zu ordnen. Eine Möglichkeit besteht darin, Ansätze des Übens nach den Zielen einzuteilen, die sie verfolgen. Bruder (2008) zum Beispiel geht von drei Formaten des Übens aus: (a) intelligentes Üben, (b) produktives bzw. vernetzendes Üben und (c) reflektierendes Üben. Intelligentes Üben zielt darauf ab, dass Lernende mathematische Zusammenhänge möglichst tief verstehen und das Üben selbst erlernen, also eine Kompetenz zum Üben (im Sinne einer Lernkompetenz) aufbauen. Produktives bzw. vernetzendes Üben hat zum Ziel, mathematische Themenfelder zu verknüpfen und größere Sinnzusammenhänge für die Lernenden zu schaffen. Reflektierendes Üben soll vor allem die Reflexion der Übenden anregen und letztlich die Selbstkompetenz (im Sinne einer Urteilskompetenz über die eigene Kompetenz) fördern. Reflektiert werden soll sowohl der Gegenstand als auch der Prozess des Übens, wozu Aspekte des intelligenten und produktiven Übens ebenfalls verwendet werden können. Die drei Übungsformate sind nicht trennscharf; hinzukommt, dass die Bezeichnungen inkonsistent verwendet werden (Leuders 2009).

Eine andere Unterscheidung trennt zwischen (a) vermischten Kopfübungen und (b) komplexen Übungen. Während vermischte Kopfübungen dazu dienen, Basiswissen durch explizite Wiederholung sozusagen wachzuhalten, sollen in komplexen Übungen bei der konkreten Anwendung Grundlagen mit geübt werden. Die dort stattfindenden Wiederholungen sind also eher implizit (Bruder 2008). Der Verweis auf explizite und implizite Wiederholung zeigt unter anderem, dass Übungen im Laufe einer mathematischen Lehr-Lerneinheit an verschiedenen Stellen mit unterschiedlicher Zielsetzung eingesetzt werden können.

Scherer und Weigand (2017) gehen einen anderen Weg und unterscheiden verschiedene didaktische Prinzipien in Übungen, verstanden als Gestaltungsregeln sowie Analyse- und Beurteilungskriterien (Scherer und Weigand 2017). So besagt etwa das Prinzip der konsequenten Wiederholung, dass regelmäßig geübt werden muss. Das Prinzip der integrierten Wiederholung zielt darauf ab, beim Üben immer 
wieder bereits gelernte Dinge aufzugreifen. Das Prinzip der Stabilisierung besteht darin, etwas wiederholt in herausfordernden und anregenden Kontexten zu üben, wenn man das zu Lernende verfügbar halten will. Prinzipien dieser Art führen in letzter Konsequenz dazu, nicht nur die Art der Aufgaben, mit denen man übt, im Blick zu haben, sondern auch die Art, wann, wie oft, in welcher Reihenfolge und Variabilität Übungen angeboten werden.

Bruder (2008) kommt zu dem Schluss, dass es aufgrund der großen Vielfalt an Übungen schwierig ist, die Qualität von Übungen an einzelnen Aufgaben bzw. Materialien und Instrumenten festzumachen. Entscheidend sei vielmehr die Art ihrer Zusammenstellung.

\section{4 Üben aus fachdidaktischer und allgemeindidaktischer Sicht}

Vergleicht man die allgemeindidaktischen mit den fachdidaktischen Überlegungen und Ansätzen des Übens, fallen Gemeinsamkeiten und Unterschiede auf. So nimmt die Mathematikdidaktik für die Schule durchaus Anleihen bei der allgemeinen Didaktik (Bruder 2008), die dann auch auf den Kontext Hochschule übertragen werden. Eine Verbindung zur allgemeinen Hochschuldidaktik wird jedoch in der fachdidaktischen Literatur kaum hergestellt. Fachdidaktische Ausführungen zum Üben konzentrieren sich tendenziell darauf, verschiedene Übeformen zu finden und einzuteilen, mit anderen Lernformen zu verbinden und in einen möglichst facettenreichen Unterricht einzubetten. Leitend scheinen die Lernpsychologie und die Metakognitionsforschung zu sein. Allgemeindidaktische Arbeiten, wie die von Brinkmann (2011, 2012), bemühen sich dagegen, den charakteristischen Kern des Übens als eine elementare Lernform herauszuarbeiten, und beziehen neben psychologischen auch historisch-rekonstruktive und phänomenologische Herangehensweisen zur Analyse und zum Verstehen des Übens mit ein.

Deutlich gemeinsam ist der allgemein- und fachdidaktischen Auseinandersetzung mit dem Üben die Konzentration auf das Können als Ergebnis. Auch die Wiederholung als notwendiger Bestandteil auf dem Weg zum Können scheint Konsens zu sein. Allgemein- wie auch fachdidaktisch betrachtet stößt ein mechanisches Üben im Sinne des Einpaukens auf Ablehnung, was sich zum Beispiel in Forderungen nach Reflexion oder nach reflexionsfördernder Aufgabengestaltung zeigt. Die Thematisierung von Erwartungen sowie Erlebnissen des Scheiterns

findet sich in allgemeindidaktischen Überlegungen als konstituierendes Moment. In fachdidaktischen Überlegungen scheint das Thema beim Üben selbst nicht dominant zu sein, findet aber in der Auseinandersetzung mit Fehlern durchaus Beachtung, zum Beispiel bei der Einbindung von individuellen (fehlerbehafteten) Präkonzepten bzw. Fehlvorstellungen (z. B. Prediger und Wittmann 2009) sowie bei der Analyse der Fehlerkultur (z. B. Schoy-Lutz 2005). 
Als unbefriedigend lassen sich Erkenntnisse zur Motivation in beiden Herangehensweisen zum Üben bezeichnen. Zwar gibt es Empfehlungen, die alle einen großen Plausibilitätscharakter haben (Bruder 2008), theoretisch begründete oder gar empirisch belegte Zusammenhänge zwischen der didaktischen Gestaltung von Übungsangeboten und unterstützenden Maßnahmen einerseits und der Bereitschaft zum Üben, Durchhaltevermögen beim Üben, Interesse am Üben oder einer motivational verankerten Übekompetenz andererseits sind jedoch nicht verfügbar. Brinkmanns (2012) Ausführungen und Deutung zum Scheitern könnten ein wesentlicher Anker sein, um diese Lücke zumindest theoretisch zu schließen.

\section{5 Üben in optes}

Als Verbundprojekt verfolgt optes das übergeordnete Ziel, die Studierenden beim Selbstlernen in mathematik-affinen Grundlagenfächern zu fördern. Studierende sollen ihre mathematischen Kenntnisse, Fähigkeiten und Fertigkeiten verbessern und gleichzeitig das Selbstlernen optimieren. Damit Studierende insbesondere zu Studienbeginn mathematisches Wissen und Können einüben und das Üben selbst lernen, stellt optes schwerpunktmäßig verschiedene materielle (elektronische) Ressourcen bereit, ergänzt durch Konzepte und Materialien, die den zusätzlichen Einsatz sozialer Ressourcen für Lehrende und Lernende unterstützen (Schmidt und Adler 2014; Derr, Hübl und Podgayetskaya 2015).

Die einzelnen Ressourcen zum Üben in optes können danach unterschieden werden, ob sie direkt dem Üben mathematischer Fähigkeiten und Fertigkeiten dienen (direkte Übeangebote) oder den Umgang mit diesen Materialien unterstützen (das Üben unterstützende Angebote).

\subsubsection{Direkte Übeangebote und das Üben unterstützende Angebote}

Inhaltlich steht hinter dem optes-Angebot ein mathematisches Curriculum (siehe Kapitel 13). Um die im Curriculum geforderten mathematischen Fähigkeiten und Fertigkeiten aufzubauen, kommen Materialen in unterschiedlichen Formen, wie (Erklär-)Texte, Übungsaufgaben, Musterlösungen oder Lernvideos, zum Einsatz. 
Der zentrale Zugang zu diesen direkten Übeangeboten erfolgt in optes thematisch geordnet über sogenannte Lernzielorientierte Kurse (LoK, siehe Kapitel 14). Sie enthalten neben Lerntexten auch Tests sowie thematische Trainings, in denen Lernende Aufgaben bearbeiten und somit üben können. Ergänzend zu den LoKs wird den Studierenden mit dem Freien Trainingsplatz ein zusätzlicher Bereich zum Üben angeboten, in welchem sie wiederholt Aufgaben ihrer Wahl (z. B. gefiltert nach Themengebiet und/oder Schwierigkeit) bearbeiten können. Der Freie Trainingsplatz bietet neben optionalen Übemöglichkeiten die Chance, Einblick in die verschiedenen Themengebiete zu erhalten und auf einfache Weise das eigene Können und Nicht-Können zu testen. Durch ein direktes Feedback werden die Studierenden in die passenden LoKs weitergeleitet, um dort die mathematischen Lerninhalte detaillierter zu bearbeiten.

Flankierend zu den direkten Übeangeboten gibt es im Rahmen von optes verschiedene das Üben unterstützende Angebote. Hierzu zählen diagnostische Tests zur Überprüfung der eigenen Fähigkeiten (siehe Kapitel 9), überfachliche Lernmodule (siehe Kapitel 16) und das E-Portfolio (siehe Kapitel 5) mit Lernzielübersicht, Lernjournal oder Fähigkeitsmatrix sowie die Lernbegleitung (siehe Kapitel 18). Zwar beeinflussen diese Angebote den Umgang der Studierenden mit den direkten Übemöglichkeiten nicht direkt, unterstützen aber den Aufbau von Selbstlern- und Übekompetenz: Sie bieten den Lernenden formative Rückmeldungen und helfen, den aktuellen Leistungsstand zu diagnostizieren, das Lernergebnis und den Lernprozess zu reflektieren und darauf aufbauend den weiteren Lernprozess zu planen.

\subsubsection{Diagnose und Zusammenspiel von Angeboten}

Studierende starten zu Beginn ihres Lernprozesses idealerweise mit einem Test zur Diagnose ihrer fachlichen und überfachlichen Kompetenzen. Auf Basis des Ergebnisses werden anschließend individuelle Lernempfehlungen generiert. Das Ergebnis und die Lernempfehlungen unterstützen Studierende darin, den eigenen Kenntnisstand einzuschätzen und mit eigenen Erwartungen abzugleichen. Gleichzeitig wird so der Umfang des Vorkurs-Programms wie auch die benötigte Lernzeit für die Studierenden eingegrenzt. Lernende können an dieser Stelle aber auch erstmals erleben zu scheitern, wenn die eigenen Erwartungen von den Ergebnissen des Tests stark abweichen. Negativen Empfindungen, die daraus resultieren können, wird in optes mit den das Üben unterstützenden Angeboten entgegengewirkt: Die Studierenden erhalten etwa Hinweise, wie sie konstruktiv mit Fehlern - die zum Lernen dazugehören - umgehen können, oder sie werden auf überfachliche Lernmodule zu Themen wie Zeit- oder Stressmanagement verwiesen. 
Ein Üben als reflexive Praxis in Momenten des Scheiterns und als ein Prozess der Selbstbildung, wie es Brinkmann $(2011,2012)$ beschreibt, erfordert allerdings ein nicht automatisiertes individuelles Feedback, was soziale Ressourcen voraussetzt. Im Falle von optes sind dies die Lernbegleiter*innen: Sie unterstützen die Studierenden, konstruktiv mit Selbsterwartungen, Enttäuschungen und dem Erleben von Negativität beim Üben umzugehen und die materiellen Ressourcen (gerade) auch in motivationalen Tiefs gezielt zu nutzen.

Ergänzend hierzu wird den Lernenden durch die Bereitstellung eines E-Portfolios die Möglichkeit geboten, beim Üben auch innezuhalten und über erzielte oder fehlende Fortschritte sowie die individuelle Arbeitsweise nachzudenken. Darüber hinaus dient diese Lernprozess-Dokumentation den Lernbegleitern als Grundlage für ihre individuelle Beratung.

\subsection{Zusammenfassung und Fazit}

Beleuchtet man das Üben als elementare Lernform im akademischen Kontext aus allgemeindidaktischer und fachdidaktischer Perspektive, werden Gemeinsamkeiten, Unterschiede sowie die Notwendigkeit deutlich, einen ganzheitlichen Blick einzunehmen, um das Üben an Hochschulen zu verstehen und zu verbessern. Am Beispiel des optes-Angebots lässt sich exemplarisch zeigen, wie sich die zum reflexiven Üben notwendigen materiellen und sozialen Ressourcen in Form von direkten Übeangeboten und das Üben unterstützenden Angeboten verzahnen und zu einem umfassenden Mathematik-Brückenkurs kombinieren lassen.

Die beschriebenen Aspekte zur Förderung des reflektierten Übens sowie die materiellen und sozialen Ressourcen sind in optes so verfügbar, dass man sie flexibel einsetzen und anpassen kann. Sie bilden ein hochschuldidaktisches Rahmenkonzept für (Online-)Mathematik-Brückenkurse, das dabei helfen soll, die Chancen und die Bedeutung zu erkennen, die das Üben gerade für Mathematik in der Studieneingangsphase hat. Dazu gehört auch, den Blick auf das Üben als mögliche Form der Persönlichkeitsentwicklung zu erweitern. Inwiefern die genannten Ressourcen und Abläufe sich auf andere Kontexte übertragen lassen, hängt letztlich von den jeweiligen Rahmenbedingungen ab. Prinzipiell möglich erscheint - auf einer abstrakteren Ebene - auch ein Transfer auf andere Übe-Gegenstände als Mathematik. Für die Mathematik speziell am Übergang zwischen Schule und Hochschule aber stellt optes ein Brückenkursangebot dar, das sich von anderen vor allem darin unterscheidet, dass direkte Übeangebote und das Üben unterstützende Angebote die beiden tragenden Säulen bilden und zu einem ganzheitlichen Konzept verzahnt worden sind. 


\section{Literatur}

Aebli, H. (2006). Zwölf Grundformen des Lehrens. Stuttgart: Klett-Cotta.

Anderson, J. R. (1993). Rules of the mind. Hillsdale, NJ: Lawrence Erlbaum Associates.

Bausch, I., Biehler, R., Bruder, R., Fischer, P. R., Hochmuth, R., Koepf, W., Schreiber, S. \& Wassong, T. (2014). Mathematische Vor-und Brückenkurse: Konzepte, Probleme und Perspektiven. Wiesbaden: Springer.

Bollnow, O. F. (1978). Vom Geist des Übens. Eine Rückbesinnung auf elementare didaktische Erfahrung. Freiburg: Herder.

Brinkmann, M. (2011). Üben. In J. Kade, W. Helsper, C. Lüders, B. Egloff, F.-O. Radtke \& W. Thole (Hrsg.), Pädagogisches Wissen. Erziehungswissenschaft in Grundbegriffen (S. 140-146). Stuttgart: Kohlhammer.

Brinkmann, M. (2012). Pädagogische Übung: Praxis und Theorie einer elementaren Lernform. Paderborn: Ferdinand Schöningh.

Bruder, R. (2008). Üben mit Konzept. Mathematik lehren, Bd. 147, 4-11.

Collins, A., Brown, J. S. \& Newman, S. E. (1989). Cognitive Apprenticeship: Teaching The Crafts of Reading, Writing and Mathematics. In L. B. Resnick (Hrsg.), Knowing, Learning and Instruction. Essays In Honour Of Robert Glaser (S. 453-494). Hillsdale, NJ: Lawrence Erlbaum Associates.

Derr, K., Hübl, R. \& Podgayetskaya, T. (2015). Formative Evaluation und Datenanalyse als Basis zur schrittweisen Optimierung eines Online-Vorkurses Mathematik. In Nistor N. \& Schirlitz S. (Hrsg.), Digitale Medien und Interdisziplinarität. Herausforderungen, Erfahrungen, Perspektiven (S. 186-196). Münster: Waxmann.

Ericsson, K. A., Krampe, R. T. \& Tesch-Römer, C. (1993). The Role of Deliberate Practice in the Acquisition of Expert Performance. Psychological Review, 100(3), 363-406.

Friedrich, H. F. \& Mandl, H. (2006). Lernstrategien: Zur Strukturierung des Forschungsfeldes. In H. F. Friedrich \& H. Mandl (Hrsg.), Handbuch Lernstrategien (S. 1-23). Göttingen: Hogrefe.

Greefrath, G., Hoever, G., Kürten, R. \& Neugebauer, C. (2015). Vorkurse und Mathematiktests zu Studienbeginn - Möglichkeiten und Grenzen. In J. Roth, T. Bauer, H. Koch \& S. Prediger (Hrsg.), Übergänge konstruktiv gestalten Ansätze für eine zielgruppenspezifische Hochschuldidaktik Mathematik (S. 19-32). Wiesbaden: Springer.

Kolb, D. A. (1984). The Process of Experiential Learning. In D. A. Kolb (Hrsg.), Experiential Learning - Experience as the Source of Learning and Development (S. 20-38). Englewood Cliffs, New Jersey: Prentice-Hall.

Leuders, T. (2009). Intelligent üben und Mathematik erleben. In T. Leuders, L. HefendehlHebeker \& H.-G. Weigand (Hrsg.), Mathemagische Momente (S. 130-143). Berlin: Cornelsen.

Meyer, H. (1987). Unterrichtsmethoden II: Praxisband. Berlin: Cornelsen.

Prange, K. (2005). Die Zeigestruktur der Erziehung. Grundriss der Operativen Pädagogik. Paderborn: Schöningh.

Prediger, S. \& Wittmann, G. (2009). Aus Fehlern lernen - (wie) ist das möglich? PM: Praxis der Mathematik in der Schule, 27, 1-8.

Prenzel, M. (2015). Institutionelle Strategien zur Verbesserung der Lehre an Hochschulen: Ein Beispiel. In Wissenschaftsrat (Hrsg.), Jahresbericht 2015 des Wissenschaftsrates (S. 50-61). Verfügbar unter https:/www.wissenschaftsrat.de/download/archiv/ Jahresbericht_2015.pdf;jsessionid=2AF6D2382C65D717A831A09614934170. delivery 1-master? blob=publicationFile\&v=3 [10.06.2020].

Rosa, H. (2016). Resonanz. Eine Soziologie der Weltbeziehung. Berlin: Suhrkamp. 
Scherer, P. \& Weigand, H.-G. (2017). Mathematikdidaktische Prinzipien. In M. Abshagen, B. Barzel, J. Kramer, T. Riecke-Baulecke, B. Rösken-Winter \& C. Selter (Hrsg.), Basiswissen Lehrerbildung: Mathematik unterrichten (S. 28-42). Seelze: Kallmeyer.

Schmidt, C. \& Adler, F. (2014). Herausforderungen an die wissenschaftliche Begleitung von E-Learning-Projekten im Hochschulverbund und konkrete Ansätze aus einem Beispielvorhaben. Interdisziplinäre Zeitschrift für Technologie und Lernen (iTeL), 1, 21 35.

Schoy-Lutz, M. (2005). Fehlerkultur im Mathematikunterricht: theoretische Grundlegung und evaluierte unterrichtspraktische Erprobung anhand der Unterrichtseinheit ,Einführung in die Satzgruppe des Pythagoras “. Hildesheim u.a.: Franzbecker.

Open Access Dieses Kapitel wird unter der Creative Commons Namensnennung 4.0 International Lizenz (http://creativecommons.org/licenses/by/4.0/deed. de) veröffentlicht, welche die Nutzung, Vervielfältigung, Bearbeitung, Verbreitung und Wiedergabe in jeglichem Medium und Format erlaubt, sofern Sie den/ die ursprünglichen Autor(en) und die Quelle ordnungsgemäß nennen, einen Link zur Creative Commons Lizenz beifügen und angeben, ob Änderungen vorgenommen wurden.

Die in diesem Kapitel enthaltenen Bilder und sonstiges Drittmaterial unterliegen ebenfalls der genannten Creative Commons Lizenz, sofern sich aus der Abbildungslegende nichts anderes ergibt. Sofern das betreffende Material nicht unter der genannten Creative Commons Lizenz steht und die betreffende Handlung nicht nach gesetzlichen Vorschriften erlaubt ist, ist für die oben aufgeführten Weiterverwendungen des Materials die Einwilligung des jeweiligen Rechteinhabers einzuholen.

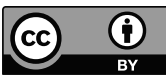

